\title{
A Cut-Resistant and Highly Restorable Graphene Foam
}

\author{
Yuan Liang, Feng Liu, Yaxi Deng, Qinhan Zhou, Zhihua Cheng, Panpan Zhang, \\ Yukun Xiao, Lingxiao Lv, Hanxue Liang, Qing Han, * Huibo Shao,* and Liangti Qu*
}

High-pressure resistant and multidirectional compressible materials enable various applications but are often hindered by structure-derived collapse and weak elasticity. Here, a super-robust graphene foam with ladder shape microstructure capable of withstanding high pressure is presented. The multioriented ladder arrays architecture of the foam, consisting of thousands of identically sized square spaces, endow it with a great deal of elastic units. It can easily bear an iterative and multidirectional pressure of $44.5 \mathrm{MPa}$ produced by a sharp blade, and may completely recover to its initial state by a load of 180000 times their own weight even under $95 \%$ strain. More importantly, the foam can also maintain structural integrity after experiencing a pressure of $2.8 \mathrm{GPa}$ through siphoning. Computational modeling of the "buckling of shells" mechanism reveals the unique ladder-shaped graphene foam contributes to the superior cut resistance and good resilience. Based on this finding, it can be widely used in cutting resistance sensors, monitoring of sea level, and the detection of oily contaminants in water delivery pipelines.
Graphene foam (GF), a 3D assembly of graphene sheets, has attracted broad attention because of its enlarged specific surface areas, good thermal and electrical conductivity useful for applications in pressure sensors, ${ }^{[1-4]}$ energy storage, ${ }^{[5-9]}$ catalysis, ${ }^{[10]}$ oil cleanup, ${ }^{[11-14]}$ and solar desalination. ${ }^{[15]}$ However, due to the weak elasticity of GF, it easily occurs structure collapse when external pressure (such as pointed compression, slight bending and stretching) is applied, thus leading to poor cyclic compressibility and high costs.

In fact, the mechanical properties are greatly related to internal microstructure of GF. The theoretical analysis and simulations deduce the mechanical mechanisms including

Y. Liang, Y. Deng, Q. Zhou, Z. Cheng, P. Zhang, Y. Xiao, L. Lv, H. Liang, Dr. Q. Han, Prof. H. Shao, Prof. L. Qu

Beijing Key Laboratory of Photoelectronic/Electrophotonic

Conversion Materials

School of Chemistry and Chemical Engineering

Beijing Institute of Technology

Beijing 100081, P. R. China

E-mail: qhan@bit.edu.cn; hbs@bit.edu.cn; lqu@bit.edu.cn

Prof. F. Liu

State Key Laboratory of Nonlinear Mechanics

Institute of Mechanics

Chinese Academy of Sciences

Beijing 100190, P. R. China

The ORCID identification number(s) for the author(s) of this article can be found under https://doi.org/10.1002/smll.201801916.

DOI: 10.1002/smll.201801916 multiscale deformation, the mesoscopic deformation, and energy dissipation, which have been proposed to explain the mechanical properties of graphene foams with various structure such as multiporous, macroporous, interconnected networks and rubber-like. ${ }^{[16-20]}$ Experimentally, a great deal of effort has been made to fabricate GF with various microstructures by using different templates. ${ }^{[4,21-30]}$ The reticulate structure GF was prepared through the chemical vapor deposition (CVD) over a Ni sponge, which displayed a compression deformation of $50 \% .{ }^{[21]}$ And the Young's modulus and compressive strength of GF present a power law dependence on density. ${ }^{[31]}$ The result shows the GF has a certainly improved elasticity after orderly construction of the internal structure, however, the harsh and complex experimental procedures are challenging to large-scale production. In this regard, polyurethane foam and melamine sponges were used as templates to fabricate GFs with network structure, ${ }^{[2,3,7,8,25-27]}$ thus leading to a maximum pressure of nearly $2 \mathrm{kPa}$. However, the pressure-resistance performance is still modest, which need further improvement. Recently, a GF with lamellar multiarch microstructure was manufactured by a bidirectional freezing process. ${ }^{[32]}$ Unfortunately, it can only be able to withstand the vertical pressure but not the horizontal.

Herein, we provide a super-robust graphene foam with a unique inner structure of multioriented microladder arrays, which is prepared by Tween-80-assisted freeze-casting (T-GF) strategy. It can bear an iterative and multidirectional cutting pressure from a sharp blade and completely recover to its initial state in each compress-release cycle even under the $95 \%$ strain. Simultaneously, after experiencing a pressure of $2.8 \mathrm{GPa}$, it can highly revert to its preliminary status through siphoning. Given those outstanding mechanical properties, the framework can be applied in cutting resistance pressure sensor, sea level monitoring, and oily contaminants (in water transmission pipeline) detecting.

The procedure for the fabrication of T-GF framework is shown in Figure 1a. Briefly, a homogeneous solution with the optimized volume ratio of T-80 and graphene oxide sheets (GOS) was freeze-dried to form 3D frozen GOS merged with T-80 (GOS/T-80), in which GOS arranged along the direction of the ice crystal arrays. These unique microstructures and macroscopic features of T-GF can be regulated by controlling the volume ratio of $\mathrm{GO}$ to $\mathrm{T}-80$ and using different molds during 


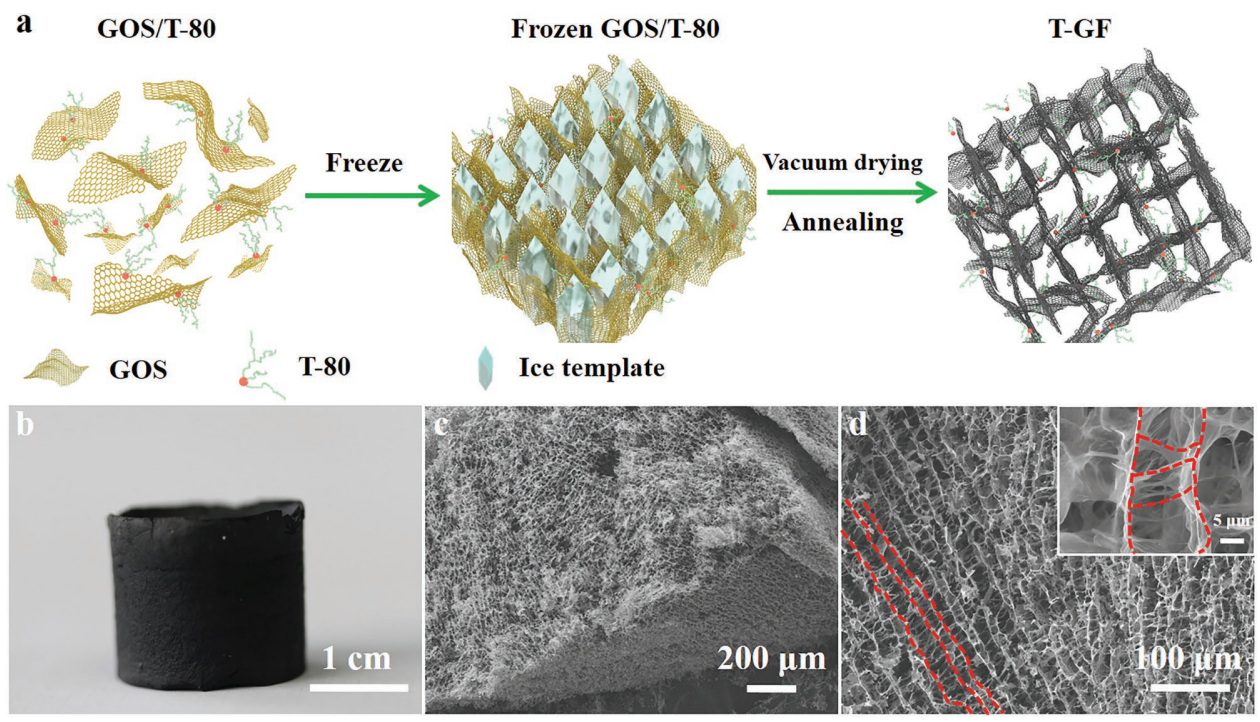

Figure 1. a) Schematic of the fabrication process of T-GF. b) Photograph of T-GF. c,d) SEM images of T-GF. Inset in (d) shows the enlarged view of the red dotted area.

the freeze-drying process (detail discussions in Figures S1 and S2, Supporting Information). Subsequent $200{ }^{\circ} \mathrm{C}$ annealing of the frozen GOS/T-80 resulted in the formation of T-GF. From the TGA (thermogravimetric analysis) of T-80, the Raman and X-ray diffraction (XRD) analysis of T-GF (Figures S3 and S4, Supporting Information), the weight ratio of resultant T-80 and reduced graphene sheets in T-GF is 3:2. The 3D foam (Figure $1 \mathrm{~b}$ ) with a density of $12 \mathrm{mg} \mathrm{cm}^{-3}$ was assembled by the multioriented ladder-shaped microstructure arrays (Figure 1c,d). Scanning electron microscopy (SEM) images in Figure $1 \mathrm{~d}$ inset and Figure S5 (Supporting Information) clearly showed that the square frames in the T-GF framework are homogenous, and $10 \pm 2 \mu \mathrm{m}$ in edge length and $14 \pm 3 \mu \mathrm{m}$ in diagonal length interconnected with each other. This unique architecture contributes to the following high compressibility in multidimension. Benefiting from its unique structure, the obtained T-GF can be compressed randomly and rapidly by our fingers as shown in Movie S1 (Supporting Information), suggesting it is firm, elastic, and strong. Meanwhile, the T-GF exhibits an excellent mechanical strength to support a heavy weight of $5 \mathrm{~kg}$ (Figure S6 and Movie S2, Supporting Information), 180000 times heavier than its own weight (about $27.3 \mathrm{mg}$ ). This loading-bearing performance is higher than any other compressible foams reported previously. ${ }^{[8,22,24,33,34]}$ Better yet, the T-GF also shows outstanding compression performance in the flat compression mode (Figure S7a, Supporting Information) and can undergo high compression condition of $99 \%$ strain (Figure S7b, Supporting Information). In addition, The T-GF presents high durability and excellent resilience, even after 500 cycles at $80 \%$ strain (Figure S7c, Supporting Information). In comparison, the original GF with randomly oriented structure (derived from direct freeze-drying of pure GO) showed poor compressibility under the second cycle (Figure S7d, Supporting Information), indicating the multioriented ladder structure has a significant effect on the rebound elasticity performance.

To prove the outstanding pressure resistance of the T-GF, a load was applied by a sharp blade (the thickness is $0.01 \mathrm{~mm}$ ), as shown in inset of Figure 2a and Figure S8a (Supporting Information). In this harsh test mode, the T-GF could endure the extreme compressions of 50,70 , and $80 \%$ and the corresponding pressures are $2.3,4.7$, and $8.6 \mathrm{MPa}$, respectively (Figure 2a). Due to the isotropic multidirectional microladder arrays in the T-GF, it could even bear high pressures from $x$, $y$, or $z$ direction with $50 \%$ compression strains (Figure $2 \mathrm{~b}$, Figure S8b and Movie S3, Supporting Information). The pressure resistances applied on T-GF can be up to 1.8, 2.8, 4.5, 8.5, and $44.5 \mathrm{MPa}$, while keeping the high compressions of 60,70 , 80\% (Movie S4, Supporting Information), 90 and 95\%, respectively (Figure 2c). After the T-GF was compressed at $50 \%$ for 600 cycles, there was only a shallow pressing mark on the foam surface due to the mechanical loss (Figure S9, Supporting Information, and Figure 2d). The SEM images (inset of Figure 2d) of the residual impressions show that there is branches collapse on the surface of T-GF, which is caused by the mechanical loss. In addition, the indention depth after 600 cycles is less than one-tenth of the T-GF height, thus indicating a strong pressure resistance, high mechanical strength, good resilience, and recyclability. The TGA result in Figure S3 (Supporting Information) indicates that the pristine T-80 mass is completely lost to atmosphere as volatiles in nitrogen environment at about $1000{ }^{\circ} \mathrm{C}$. The obtained foam (T-GF-1000, Figure 2e) demonstrated remarkable cut resistant property with max. $80 \%$ compression. It also has the multioriented ladder microstructure (inset of Figure 2e) and good conductivity of $18 \mathrm{~S} \mathrm{~m}^{-1}$ compared to the previous work (Table S1, Supporting Information). In contrast, under the same test mode, the directly freeze-dried GF with the randomly oriented porous structure was cut off in the second compression cycle with strain of less than 50\% (Figure S10a, Supporting Information). This comparison result demonstrates the ladder microstructure contributes to the cut resistance. The other samples with different amount of T-80 are cracked in the third cycle at the strain of $30 \%$ (Figure S10b-d, Supporting Information). Some representative foams prepared according to 


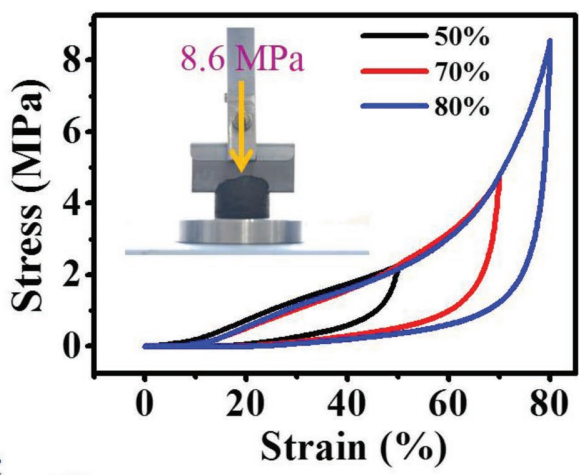

C

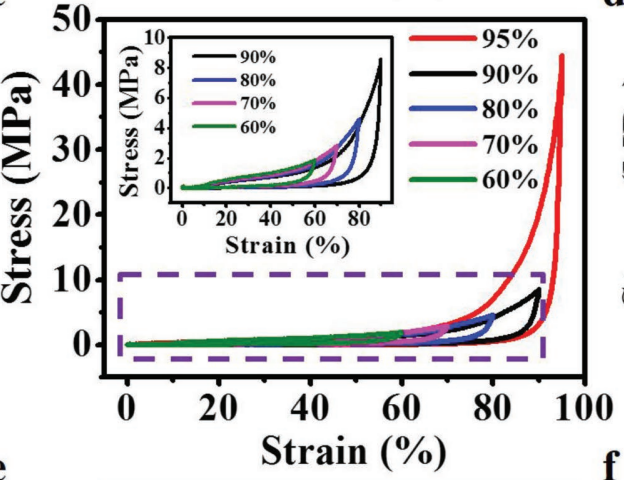

$\mathbf{e}$

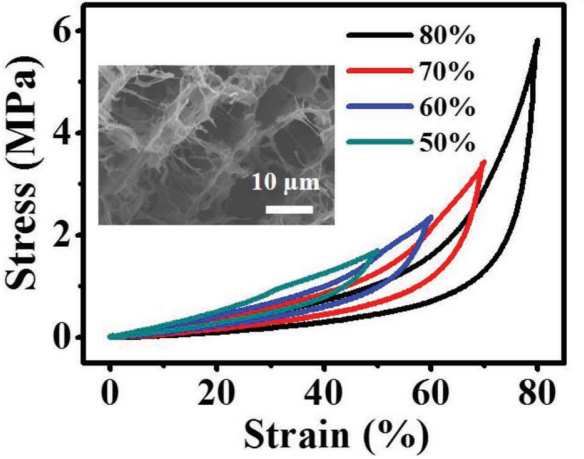

d b
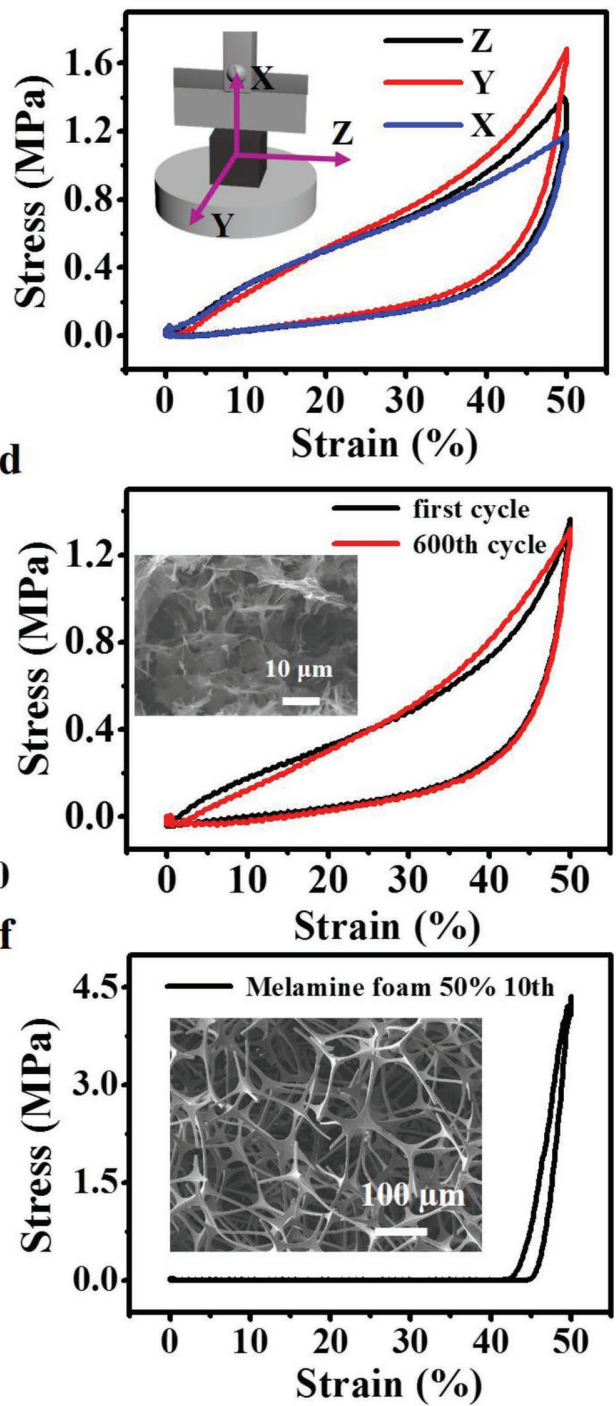

Figure 2. a) Stress-strain curves of T-GF at different strains of 50,70 , and $80 \%$, respectively. Inset: schematic diagram of the blade test mode. b) Stress-strain curves of T-GF at the strain of $50 \%$ applied by a force in the direction of $x, y$, and $z$ axes. Inset: schematic diagram of the cutting directions. c) Cutting resistance of T-GF at different strains of $60,70,80,90$, and $95 \%$, respectively. d) Fatigue durability of T-GF at the strain of $50 \%$ before and after 600 cycles. Inset: SEM image of the shallow pressing mark. e) The stress-strain curves of T-GF-1000 at strain of 50, 60, 70, and 80\%. Inset: SEM image of T-GF-1000. f) The tenth stress-strain curve of the melamine foam at the strain of 50\%. Inset is the SEM image of the melamine foam. $c-f)$ The tests are conducted in the sharp blade mode as displayed in the inset of (b).

the reported references will crack no more than $50 \%$ under the blade testing mode (Figure S11, Supporting Information). Moreover, the commercial multihole melamine sponge exhibited no toleration to the blade at the strain of $50 \%$ after ten cycles, its structure completely collapsed (Figure 2f). These results further suggest that the large elastic deformation and high pressure without structural damage of T-GF is closely related to its multioriented microladder arrays architecture. Based on this excellent mechanical property of blade-cutting resistance, the T-GF has been applied in harsh pressure sensor (Figure S12, Supporting Information), which presented stable response signals of resistance variation at the strain of 30,50 , and $80 \%$ with a maximum pressure of up to $5 \mathrm{MPa}$.
To understand the origin of this superelasticity under the blade cutting, the mechanical simulations based on finite element method (FEM) are performed. A square cellular structure composed by replicated thin shells (the upper inset in Figure 3a) is established to simulate the ladder-shape microstructure formed in T-GF (Figure 1d). Considering the thickness of the blade is close to the characteristic length scale of the microstructure, a constant velocity is applied on the middle shell of the upper surface to simulate the blade cutting. Since it is nonuniform mechanical loading, strain energy is used to estimate its deformation degree. The evolution of strain energy with respect to loading time is shown in Figure 3a, a peak at the beginning is found, which could be attributed to the 
a

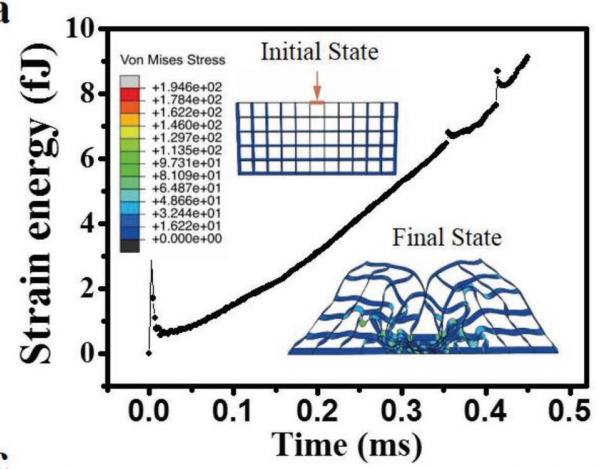

b
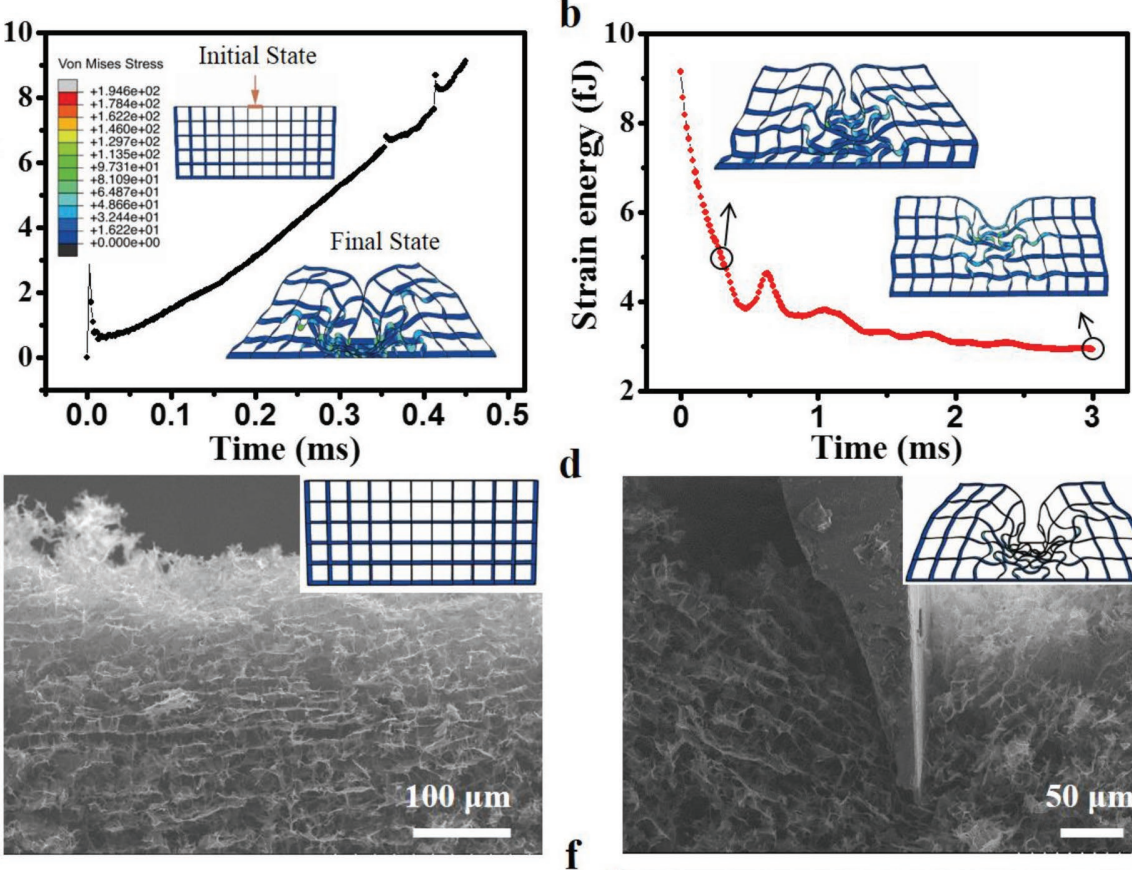

d
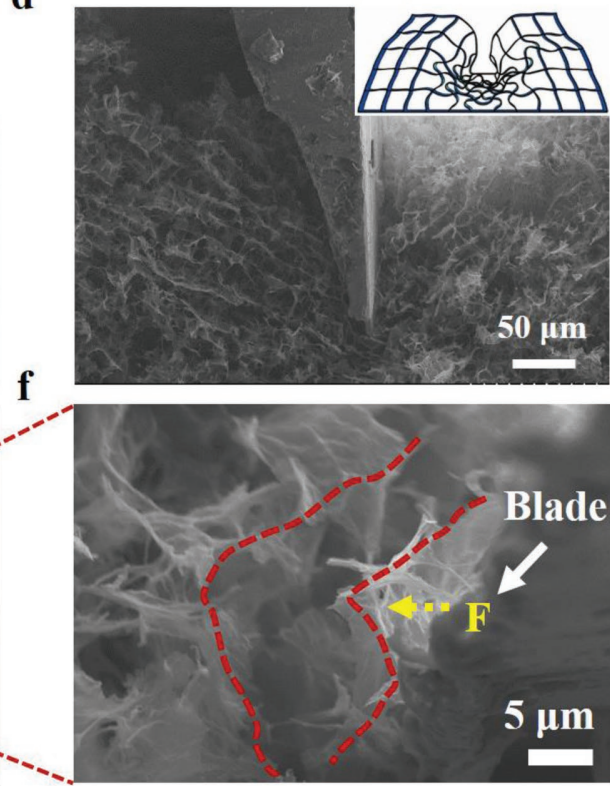

Figure 3. a) The process of using FEM to simulate the cutting process: a relation curve about the evolution of strain energy with loading time under a load applied on the middle shell of the upper surface with a velocity of $100 \mathrm{~mm} \mathrm{~s}^{-1}$ and the simulation lasts for $0.45 \mathrm{~ms}$. Insets: the left-upper part of curve is the initial state of the sample and the color represents the magnitude of Von Mises stress (the unit is MPa); the right-lower part is the final state of the sample. b) The spring-back stimulation process. Insets: the strain states of every structural unit at given time (black circles). c,d) The comparison of the FEM simulation results with the experimental results before and after the blade cutting. e,f) The deformation of an individual square in front of the cutting blade.

initial extreme deformation concentration around the loading shell. As the mechanical response of the sample catching up, the strain energy returns to a lower level. After that, the strain energy increases with the loading shell moving down. When the simulation ends up, the loading shell drops $45 \mu \mathrm{m}$ that is $90 \%$ of its initial height. Then, the constraint to the loading shell is removed to observe the spring-back process. The whole simulation process lasts for $3 \mathrm{~ms}$, and about 70\% strain energy is released (Figure 3b). There is a good agreement between the observed structure evolution in our experiments and simulations (Figure 3c,d), based on which the mechanism of the superelasticity for the blade cutting could be understood. A typical image in front of the cutting blade is given in Figure 3e,f. Despite the real structure is more complicated than the theoretical model used in the finite element simulation, it can be seen that an apparent buckling occurs for a single square unit in front of the cutting blade, which clearly convey the "buckling of shells" mechanism. Under loading, the sample deforms via the buckling of thin shells, and then springs back to its original shape within a short time. Thus, graphene foam with the ladder shape microstructure has a remarkable effect to tolerate a large local deformation without microstructure destruction.

To further explore the ultimate bearing capacity, the T-GF is subjected to an extremely high pressure of $2.8 \mathrm{GPa}$ by commercial table compressing machine. After that, the 3D foam with a height of $1.4 \mathrm{~cm}$ is transformed into a film with a thickness of only $1 \mathrm{~mm}$ (Figure 4a,b). Excitingly, when the compressed T-GF film absorbed liquids (water, ethanol, or oils, Figure 4c and Figure S13, Supporting Information), it can almost rebound into its initial 3D state, due to the siphon and hydrophilia (Figures S14 and S15, Supporting Information) that the liquid filled in the trapezoid-shaped channel of T-GF. Contrast to the regular square frames in the initial state (see Figure $4 d$ ), the graphene flakes are densely stacked in the compressed state (Figure 4e) suggesting the adhesion energy could overcome the elastic energy in this case and thus prevents the 
a
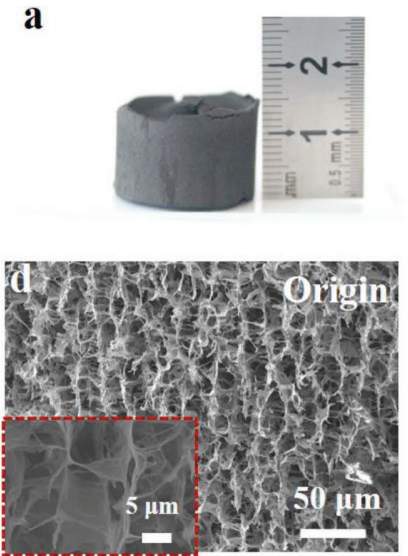

g

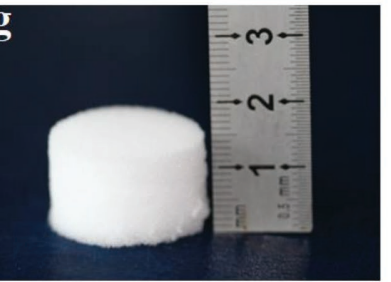

b
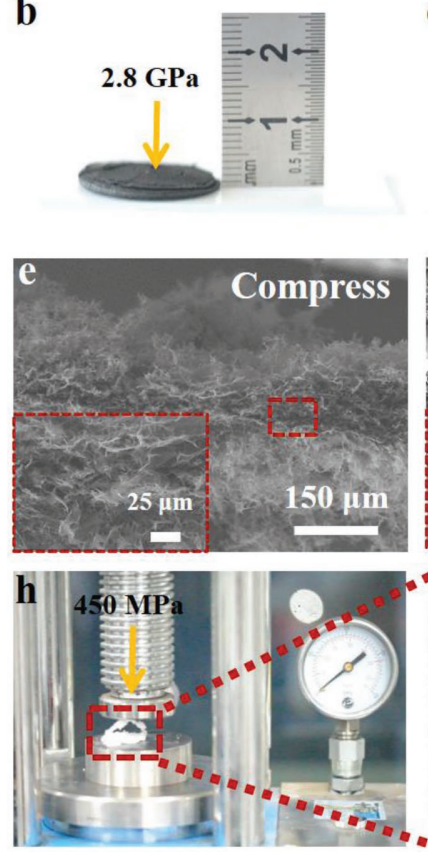

c
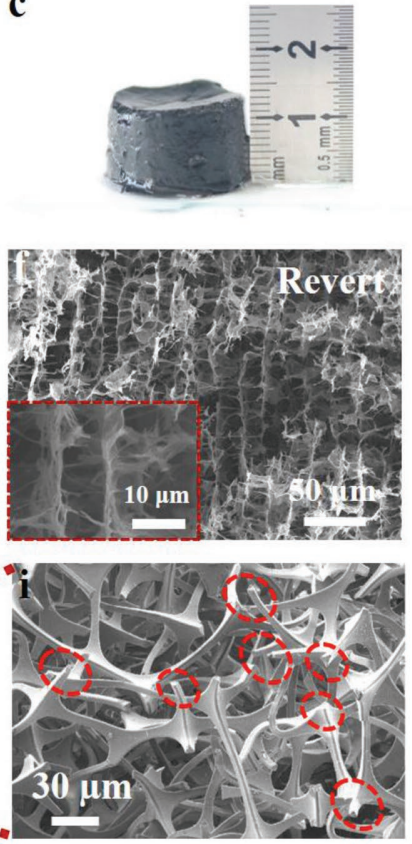

Figure 4. a-c) The recovery capability of T-GF. Photograph of a) initial state of T-GF, b) the state of T-GF after experiencing a high pressure of 2.8 GPa, and c) the restored state of T-GF absorbed water. $d-f$ ) SEM images of a) initial, b) compressed state, and c) restored state. Inset is the enlarged SEM. g) The initial state of commercial melamine foam. h,i) The photograph and SEM image of the collapsed melamine foam after suffering a $450 \mathrm{MPa}$ pressure.

recovery of the system. It was found that the microstructure in the T-GF maintains very well (Figure $4 \mathrm{f}$ and inset) after suffering from this high pressure, in relative to the original morphology (Figure 4d). Thus, the plastic deformation mechanism at very high applied pressure is supposed to originate from the great adhesion energy between graphene sheets. It is believed that there is an energy barrier between the compressed state and the initial state, and for the compressed state the adhesion energy dominates and thus prevents the recovery of the system. In addition, T-GF shows a spring-back pressure of $9 \mathrm{kPa}$ in the first cycle after absorbing water and keeps stability from second to tenth cycle (Figure S16, Supporting Information). In comparison, commercial melamine foam (Figure $4 \mathrm{~g}$ ) collapses into powder and its internal structure is destroyed at pressure of $450 \mathrm{MPa}$ for only once (Figure 4h,i and Movie S5, Supporting Information), further revealing the excellent recoverability of T-GF.

Even more encouraging, the resilience pressure of the compression-absorption-recover process can be utilized to lift a heavy object. As shown in Figure 5a,b, a compressed T-GF film of $3 \mathrm{mg}$ is able to lift comfortably a $1 \mathrm{~g}$ load that is about 333 times of its weight after absorbing water (Figure 5a,b and Movie S6, Supporting Information). In addition, the compression-absorption-recover effect combined with $0.4 \mathrm{~S} \mathrm{~m}^{-1}$ electrical conductivity of the T-GF can be used to monitor the change of water level. As shown in Figure $5 \mathrm{c}$, the compressed T-GF film is placed between two copper mesh electrodes in noncontact mode. Once the rising water level reached the bottom of the compressed T-GF film, it would absorb water and volume expand to contact with the top electrode. Consequently, the circuit was closed and a light-emitting diode (LED) was turned on as a warning sign (Figure S17 and Movie S7, Supporting Information). Apart from that, the T-GF could be used for detection of the leakage of oily contaminants in the drain water piping (Figure $5 \mathrm{~d}$ ) by regulation of T-GF properties such as hydrophobicity. Given that, a functional T-GF (named as FT-GF) with hydrophobic and lipophilic property (Figures S18 and S19 and Movie S8, Supporting Information) was obtained by adding traces of hydrophobic $1 \mathrm{H}, 1 \mathrm{H}, 2 \mathrm{H}, 2 \mathrm{H}$-perfluorooctyltriethoxysilane in the precursor of GOS/T-80 solution. To demonstrate this application, the FT-GF with the similar electrical conductivity with T-GF was compressed into FT-GF film, which was punched a pore in the center to make water flow successfully (Figure $5 \mathrm{~d}$ ). When water flew through the sample, the FT-GF film did not rebound and the circuit was in open state. Once the oil (e.g., octane) flew through the film, it would swell and connect the two electrodes together. Finally, the LED in the circuit was lit up as a detection signal (see the details in Movie S9, Supporting Information).

In summary, we have demonstrated a robust graphene foam with multioriental microladder structure induced by T- 80 assistant freeze-casting process. This unique structure endows the T-GF with outstanding compressing elasticity, excellent cutting resistance, and highly recoverable property. All the combined characters make the T-GF possible to apply in cutting resistance pressure sensor, sea level monitoring, and detection of oily contaminants in water transmission pipeline, which opens up numerous new opportunities for a range of application areas. Furthermore, other functional materials can be readily merged 

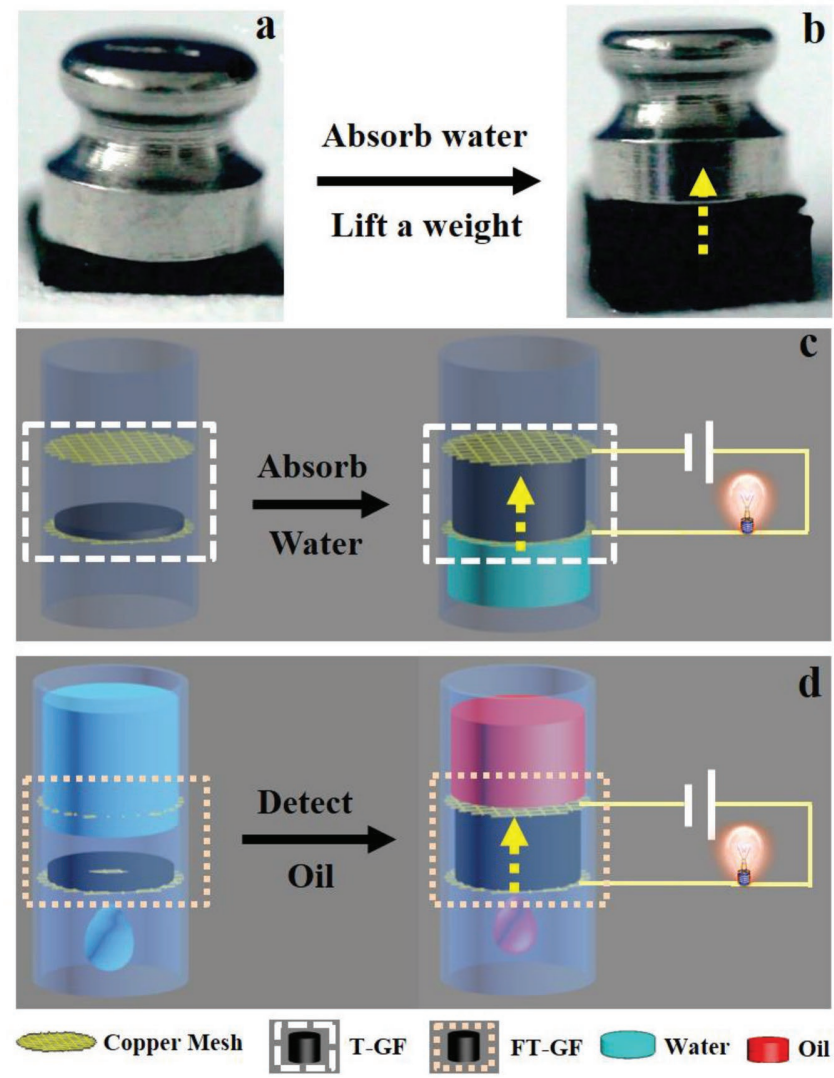

Figure 5. a,b) The procedure of lifting a weight of 333 times its own weight after absorbing water. c) The process of monitoring the change of water level. d) The function of detecting the oil leaked in water transmission pipeline.

into this unique structure, providing even the broader foreground for applications.

\section{Supporting Information}

Supporting Information is available from the Wiley Online Library or from the author.

\section{Acknowledgements}

This work was supported by the National Key R\&D Program of China (2017YFB1104300 and 2016YFA0200200), National Science Foundation of China (No. 51673026, 21674056, 21773007, 21575014, 11602272 , and 21173023), Beijing Natural Science Foundation (2152028 and 2184122), Beijing Municipal Science and Technology Commission (Z161100002116022), 111 Project 807012, the Fundamental Research Funds for the Central Universities (2018CX01017), Beijing Institute of Technology Research Fund Program for Young Scholars, and the Strategic Priority Research Program of the Chinese Academy of Sciences (No. XDB22040503). The authors also acknowledge the project of State Key Laboratory of Explosion Science and Technology (Beijing Institute of Technology). The project number is YBKT18-03.

\section{Conflict of Interest}

The authors declare no conflict of interest.

\section{Keywords}

cut resistance, elastic resilience, ladder-shaped graphene foam, oily pollutants detecting, sea level monitoring

Received: May 19, 2018

Revised: June 11, 2018

Published online:

[1] L. Lv, P. Zhang, T. Xu, L. Qu, ACS Appl. Mater. Interfaces 2017, 9, 22885.

[2] Y. A. Samad, Y. Li, A. Schiffer, S. M. Alhassan, K. Liao, Small 2015, 11,2380

[3] H.-B. Yao, J. Ge, C.-F. Wang, X. Wang, W. Hu, Z.-J. Zheng, Y. Ni, S.-H. Yu, Adv. Mater. 2013, 25, 6692.

[4] Y. Si, X. Wang, C. Yan, L. Yang, J. Yu, B. Ding, Adv. Mater. 2016, 28, 9512.

[5] Y. Zhao, J. Liu, Y. Hu, H. Cheng, C. Hu, C. Jiang, L. Jiang, A. Cao, L. Qu, Adv. Mater. 2013, 25, 591.

[6] H. Chen, C. Chen, Y. Liu, X. Zhao, N. Ananth, B. Zheng, L. Peng, T. Huang, W. Gao, C. Gao, Adv. Energy Mater. 2017, 7, 1700051.

[7] X. Wang, L. Lv, Z. Cheng, J. Gao, L. Dong, C. Hu, L. Qu, Adv. Energy Mater. 2016, 6, 1502100.

[8] K. Xiao, L.-X. Ding, G. Liu, H. Chen, S. Wang, H. Wang, Adv. Mater. 2016, 28, 5997.

[9] Y. Jiang, H. Shao, C. Li, T. Xu, Y. Zhao, G. Shi, L. Jiang, L. Qu, Adv. Mater. 2016, 28, 10287.

[10] Y. Zhao, C. Hu, L. Song, L. Wang, G. Shi, L. Dai, L. Qu, Energy Environ. Sci. 2014, 7, 1913.

[11] H. Zhu, D. Chen, W. An, N. Li, Q. Xu, H. Li, J. He, J. Lu, Small 2015, 11, 5222.

[12] H. Bi, X. Xie, K. Yin, Y. Zhou, S. Wan, L. He, F. Xu, F. Banhart, L. Sun, R. S. Ruoff, Adv. Funct. Mater. 2012, 22, 4421.

[13] Y. Zhao, C. Hu, Y. Hu, H. Cheng, G. Shi, L. Qu, Angew. Chem., Int. Ed. 2012, 51, 11371.

[14] Z. Niu, J. Chen, H. H. Hng, J. Ma, X. Chen, Adv. Mater. 2012, 24, 4144.

[15] P. Zhang, J. Li, L. Lv, Y. Zhao, L. Qu, ACS Nano 2017, 11, 5087.

[16] C. Wang, D. Pan, S. Chen, Carbon 2018, 132, 641.

[17] D. Pan, C. Wang, T.-C. Wang, Y. Yao, ACS Nano 2017, 11, 8988.

[18] P. Nautiyal, B. Boesl, A. Agarwal, Carbon 2018, 132, 59.

[19] A. Nieto, B. Boesl, A. Agarwal, Carbon 2015, 85, 299.

[20] C. Wang, C. Zhang, S. Chen, Carbon 2016, 109, 666.

[21] Z. Chen, W. Ren, L. Gao, B. Liu, S. Pei, H.-M. Cheng, Nat. Mater. 2011, 10, 424.

[22] J. Sha, C. Gao, S.-K. Lee, Y. Li, N. Zhao, J. M. Tour, ACS Nano 2016, $10,1411$.

[23] F. Yavari, Z. Chen, A. V. Thomas, W. Ren, H.-M. Cheng, N. Koratkar, Sci. Rep. 2011, 1, 166.

[24] H. Bi, I.-W. Chen, T. Lin, F. Huang, Adv. Mater. 2015, 27, 5943.

[25] B. Shen, Y. Li, W. Zhai, W. Zheng, ACS Appl. Mater. Interfaces 2016, 8, 8050.

[26] X. Du, H.-Y. Liu, Y.-W. Mai, ACS Nano 2016, 10, 453.

[27] C. Wu, X. Huang, X. Wu, R. Qian, P. Jiang, Adv. Mater. 2013, 25, 5658.

[28] B. Yao, J. Chen, L. Huang, Q. Zhou, G. Shi, Adv. Mater. 2016, 28, 1623.

[29] L. Qiu, J. Z. Liu, S. L. Y. Chang, Y. Wu, D. Li, Nat. Commun. 2012, 3, 1241.

[30] F. Zhao, L. Wang, Y. Zhao, L. Qu, L. Dai, Adv. Mater. 2017, 29, 1604972. 
[31] W. Park, X. Li, N. Mandal, X. Ruan, Y. P. Chen, APL Mater. 2017, 5, 036102.

[32] H.-L. Gao, Y.-B. Zhu, L.-B. Mao, F.-C. Wang, X.-S. Luo, Y.-Y. Liu, Y. Lu, Z. Pan, J. Ge, W. Shen, Y.-R. Zheng, L. Xu, L.-J. Wang, W.-H. Xu, H.-A. Wu, S.-H. Yu, Nat. Commun. 2016, 7, 12920.
[33] L. Lv, P. Zhang, H. Cheng, Y. Zhao, Z. Zhang, G. Shi, L. Qu, Small 2016, 12, 3229

[34] P. S. Owuor, T. Tsafack, H. Y. Hwang, O.-K. Park, S. Ozden, S. Bhowmick, S. A. S. Amanulla, R. Vajtai, J. Lou, C. S. Tiwary, P. M. Ajayan, ACS Nano 2017, 11, 806. 